\title{
Observability for PMU-Based Monitoring of Nonlinear Power System Dynamics
}

\author{
Guanqun Wang, Chen-Ching Liu* \\ Washington State University, USA \\ *Also University College Dublin (UCD), Ireland \\ gwang@eecs.wsu.edu \\ liu@eecs.wsu.edu
}

\author{
Navin Bhatt, Evangelos Farantatos, Kai Sun* \\ Electric Power Research Institute, USA \\ *Also University of Tennessee, USA \\ nbhatt@epri.com \\ efarantatos@epri.com \\ ksun@epri.com
}

\begin{abstract}
PMUs placed at different buses enable dynamic monitoring of a power system. The question is whether those limited PMU measurements can provide sufficient information. In this research, observability analysis is used to answer this question. Different observability concepts together with their related state monitoring applications are discussed in this paper. Nonlinear dynamic observability based on Lie derivatives is applied to the PMU-based dynamic monitoring of power system. The smallest singular value of the observability matrix serves as an observability index. A 3-bus system is used to illustrate the observability concepts presented in this paper. The concepts of observability are clarified and the nonlinear observability analysis provides a basis for PMU-based on-line wide area monitoring and control to improve the dynamic performance of power systems.
\end{abstract}

Keywords: Observability Analysis, State Estimation, Phasor Measurement Unit (PMU), Lie Derivative

\section{Introduction}

The advent of phasor measurement unit (PMU) provides an opportunity for wide area monitoring and control of the power grid due to its fast sampling rate and timesynchronization capability. Although the number of PMUs installed on the grid has increased significantly in recent years, the total number of monitored points is still modest relative to the large number of buses in an interconnected grid [1]. When the states of the system are to be estimated and reconstructed, it is always questionable whether these PMU measurements contain sufficient information for estimation of the entire set of

G. Wang and C. C. Liu are with Washington State University, USA, C. C. Liu is also with UCD, Ireland. N. Bhatt, E. Farantatos and K. Sun are with Electric Power Research Institute (EPRI). K. Sun is also with University of Tennessee, USA. G. Wang and C. C. Liu gratefully acknowledge the financial support of EPRI. states and how reliable the results are [2-4]. This is an issue of observability.

Observability analysis is a crucial task in model-based state monitoring and estimation. The concepts of observability are defined for different contexts and in different ways [2-7]. Traditionally, for the static case, a power system is considered observable if the set of measurements is sufficient to solve static state estimation problem [5-6]. Particularly, for static state estimation with measurements provided by PMUs, the observability problem can be formulated as graph covering problem [23]. If the complete set of bus voltage phasors can be determined by the number and location of the PMUs, the system with this PMU placement scenario is called topological observability, and the state estimation problem becomes linear [2]. When dynamic states are required for monitoring and control, it is necessary to apply the dynamic observability concept that incorporates the knowledge of system dynamics. Reference [7] presents a general algorithm of nonlinear observability analysis with application to model-based diagnostics and sensor selection in three-phase induction motors. In [4], the observability is derived from a differential-algebraic equation (DAE) model of power system and can be tracked along system dynamic trajectories.

This paper clarifies different observability concepts for static and dynamic models. The nonlinear dynamic observability based on Lie derivative is applied to PMUbased monitoring of system dynamics. An index based on the singular value of observability matrix is obtained to quantify the level of observability for dynamic state calculation with partial PMU measurements. A 3-bus system is used to demonstrate the observability concepts presented in this paper. For the dynamic case, the observability matrix is derived through system differential equations and observability index is obtained along the reconstructed trajectories of system states. By comparing different placements of the PMUs, the results indicate that 
when the number of PMUs is small, the system indeed has a lower level of dynamic observability, which is expected. With a higher level of observability, the dynamic states reconstructed from PMU measurement are more reliable.

In the remaining of this paper, Section 2 provides a discussion of static observability, while Section 3 gives the concept of topological observability. Section 4 includes details regarding dynamic linear and nonlinear observability and the theoretical foundation by Lie derivative. In Section 5, the PMU-based dynamic monitoring problem is described together with the justification for nonlinear observability to be applied to this problem. A case study with a 3-bus system is presented in Section 6, followed by the concluding remarks.

\section{Static Observability}

The power system static state estimation problem can be formulated as [8]

$$
z=h(x)+\varepsilon
$$

in which $x$ is the state vector representing bus voltages and angles; $z$ is the measurement vector that includes the real power of buses and lines; $h$ is the vector of measurement functions; $\varepsilon$ is the vector of measurement errors.

In static state estimation, a network is said to be observable if any flow in the network can be observed by some sort of indication in the set of measurements [5]. For simplicity, give the measurement equation by DC power flow model

$$
z=H \theta+\varepsilon
$$

in which $\theta$ is the state vector of the bus angles and $H$ is the measurement matrix, formed by line reactance $1 / x$. By the least-squared algorithm for static state estimation, $\theta$ can be obtained by

$$
\theta^{*}=\left(H^{T} H\right)^{-1} H^{T} Z
$$

Therefore, if $H^{T} H$ is nonsingular, that is, the rank of $\mathrm{H}$ equals to H's column number, the system is observable. The observability for static state estimation is different depending on whether the number of measurements is greater than (or equal to) or less than the number of system states. Equation (3) applies to the case when the number of measurements is greater than or equal to the number of states. This solution also corresponds to the maximum likelihood estimate for jointly Gaussian white noises of the measurement errors. Therefore, it is physically meaningful. For an observable system, the ratio of the number of measurements to the number of states is defined as measurement redundancy, which, in most cases, is in the range 1.7-2.2. A critical measurement is a non-redundant measurement, i.e., a measurement that results in network unobservability when it is removed [9].

If the number of measurements is lower than that of the state variables, it is possible to obtain a minimum norm estimate based on the available measurements. However, this minimum norm estimate does not have strong evidences as a physically meaningful solution. If a network is unobservable, it is still useful to know which portion can be estimated, i.e., to determine the observable islands [10].

\section{Topological Observability}

For static state estimation with measurements provided by PMUs, however, it is possible to observe beyond the PMU buses [2]. A PMU may have multiple input channels for voltage and current signals. Since each bus of a power system has only a limited number of neighbors, the PMU installed at a specific bus is capable of measuring not only the bus voltage phasor, but also the current phasors along all lines incident with the bus. As a result, in addition to the voltage phasor at this bus, the voltage phasors of all neighboring buses can also be computed, hence they also become observable.

If the complete set of bus voltage phasors can be determined by the number and location of PMUs, the system with this PMU placement scenario is called the system topological observability [11]. For a topologically observable network with only PMU measurements, the static state estimation becomes linear, as shown in the following equation

$$
\left(\begin{array}{c}
z_{V} \\
z_{C}
\end{array}\right)=\left(\begin{array}{cc}
I & 0 \\
Y_{C L 1} & Y_{C L 2}
\end{array}\right)\left(\begin{array}{c}
\dot{V}_{L 1} \\
\dot{V}_{L 2}
\end{array}\right)+\left(\begin{array}{c}
\varepsilon_{V} \\
\varepsilon_{C}
\end{array}\right)
$$

in which $\dot{V}_{L 1}, \dot{V}_{L 2}$ are state vectors representing the bus voltage phasor at buses with and without PMU measurements; $I$ is identical matrix. $Y_{C L 1}$ and $Y_{C L 2}$ are the measurement matrices with the network admittances; $z_{V}$ and $z_{C}$ are the measurement vectors with the bus voltages and line currents; $\varepsilon_{V}$ and $\varepsilon_{C}$ are the vector of measurement errors. 
As a result, the observation problem becomes a graph covering problem. The optimal placement problem of PMUs for complete observability of the network can be determined by the following integer linear programming

$$
\begin{array}{cc}
\min & C^{T} x_{i} \\
\text { s.t. } & A X \geq b
\end{array}
$$

in which $C$ is the cost vector for installation of PMUs; $X$ is a binary vector which is 1 for presence or 0 for absence of PMUs at buses; $A$ is a binary matrix mapping nonzero elements of the node admittance matrix to ones and $b$ is the vector of ones.

According to [12], a minimum number of $1 / 5$ to $1 / 4$ of the system buses to be installed with PMUs should ensure the complete observability of the system, which is different from static observability case.

As a result, the static observability and topological observability determination in power systems are consistent with the solvability of the state estimation problem. The system is considered observable if the states can be uniquely determined from the measurements.

\section{Dynamic Observability}

The static state estimation accounts only for the static system variables without the dynamic system states. For control techniques that require information about these dynamic system states, the static state estimation methods and corresponding observability concepts is not sufficient.

For a dynamic system model in differential equations

$$
\begin{gathered}
\dot{x}(t)=f(x(t))+u(x(t)) \\
z=h(x(t))
\end{gathered}
$$

where $x$ and $z$ represent state vector and measurement vector, $f, u$ and $h$ are state function vector, control function vector and measurement function vector. The system is said to be observable if, for any possible sequence of state and control vectors, the current state can be determined in finite time using the outputs [13]. In other words, for an observable system, it is possible to use the system's measurements to determine the states of the dynamic system. If a system is not observable, the current values of some of its states may not be determined through the output values. The definition of dynamic observability is established using Lie derivative.

\subsection{Lie Derivative}

Let $f: \mathbb{R}^{n} \rightarrow \mathbb{R}^{n}$ be a vector field in $\mathbb{R}^{n}$, that is

$$
f=\left[\begin{array}{c}
f_{1}(x) \\
\ldots \\
f_{n}(x)
\end{array}\right]
$$

Let $h: \mathbb{R}^{n} \rightarrow \mathbb{R}$ be a scalar function, that is

$$
h(x)=\left[\begin{array}{llll}
h_{1}(x) & h_{2}(x) & \cdots & h_{p}(x)
\end{array}\right]^{T}
$$

Then the Lie derivative of $h$ with respect to $f$ is

$$
L_{f} h=\nabla h \cdot f(x)=\frac{\partial h}{\partial x} f=\sum_{i=1}^{n} \frac{\partial h}{\partial x_{i}} f_{i}
$$

The higher-order Lie derivatives can also be defined. By definition, the zero-order Lie derivative of $h$ is

$$
L_{f}^{0} h=h
$$

and the k-order derivative could be calculated by repeated derivatives, i.e.,

$$
L_{f}^{k} h=\frac{\partial\left(L_{f}^{k-1} h\right)}{\partial x} \cdot f
$$

\subsection{Observability Criterion}

For a nonlinear dynamic system defined in (7) and (8), it is said to be locally observable at $x_{t}=x(t)$ if the matrix

$$
O\left(x_{t}\right)=\left.\frac{\partial l(x)}{\partial x}\right|_{x=x_{t}}
$$

has a full rank [14]. $O(x)$ is called the observability matrix, in which

$$
l\left(x_{t}\right)=\left[\begin{array}{c}
L_{f}^{0} h_{1}(x) \\
\cdots \\
L_{f}^{0} h_{p}(x) \\
L_{f}^{1} h_{1}(x) \\
\cdots \\
L_{f}^{n-1} h_{p}(x)
\end{array}\right]
$$


The rank condition of (13) can be checked by the singular value decomposition method [15]. Since the observability property is local, the smallest singular value of $O(x)$ will change along the trajectory of $x(t)$. Furthermore, the smallest singular value of $O(x)$ can be used as an index to compare the level of observability of the system with different outputs [15].

The calculation of observability for nonlinear systems is a complex task, especially when the order of system is high. However, for time-invariant linear systems in the state space representation, the criteria for checking the observability is simplified. Consider the linear dynamic system

$$
\begin{aligned}
& \dot{x}=f(x(t))=A x(t) \\
& z=h(x(t))=M x(t)
\end{aligned}
$$

It is well known that the observability matrix in (13) is in the form of

$$
O=\left[\begin{array}{c}
M \\
M A \\
\cdots \\
M A^{n-1}
\end{array}\right]
$$

The system is observable if the observability matrix is nonsingular, based on the standard linear system theory [16].

\section{PMU-based monitoring of power system dynamics}

Besides static state variables, i.e., bus voltages and angles, it is important to monitor power system dynamic states such as the rotor speed to ensure efficient and reliable operation and control of the grid. Since PMU data can be acquired to approximate the continuous time waveforms with high accuracy and synchronized time stamps, their installation can considerably improve monitoring of the system dynamics. The dynamics of the power system need to be modeled by a set of differential equations as (7) and the PMU-measured waveform can be treated as (8). The dynamic states can be reconstructed from the measurements using numerical integration method, i.e., implicit integration with trapezoidal rule [1] or dynamic state estimation method, i.e., extended Kalman filter method [17].

With these state calculation methods, the full states of the system are solvable no matter how many measurements are available on the system. However, the accuracy of monitoring is questionable when the number of measurements is small compared to the number of states. As a result, the static observability and topological observability are not appropriate in this case while the theory of dynamic observability can be applied to the PMU-based dynamic monitoring problem. The observability index serves as a metric for quality of the reconstructed dynamic states based on snapshots of PMU measurements.

\section{Example}

A 3-bus system with lossless transmission lines is used to illustrate the observability concepts, as shown in Fig. 1.

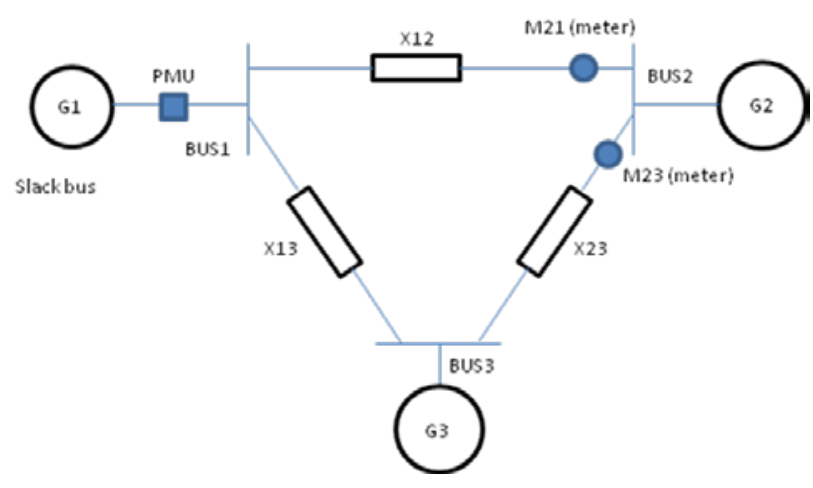

Figure 1 A simplified 3-bus system

\subsection{Static Observability}

Assume that two meters are available on line 1-2 and 2-3, measuring the line power flow. The measurement equation based on DC power flow is

$$
\left[\begin{array}{l}
z_{21} \\
z_{23}
\end{array}\right]=\left[\begin{array}{l}
P_{21} \\
P_{23}
\end{array}\right]=\left[\begin{array}{cc}
B_{12} & 0 \\
B_{23} & -B_{23}
\end{array}\right]\left[\begin{array}{l}
\theta_{2} \\
\theta_{3}
\end{array}\right]
$$

where $B$ represents line susceptance. The rank of measurement matrix is 2. As a result, the system is observable and the static estimation problem is solvable.

\subsection{Topological Observability}

Assume there is only one PMU which is placed at bus 1 for measurement of the bus voltage phasor and line current phasor incident with bus 1 . The voltage phasors at bus 2 and 3 can be calculated and the system is observable. The state estimation problem an be represented by 


$$
\left(\begin{array}{c}
z_{V} \\
z_{C}
\end{array}\right)=\left(\begin{array}{l}
\dot{V}_{1} \\
\dot{I}_{12} \\
\dot{I}_{13}
\end{array}\right)=\left(\begin{array}{ccc}
1 & 0 & 0 \\
B_{12} & -B_{12} & 0 \\
B_{13} & 0 & -B_{13}
\end{array}\right)\left(\begin{array}{c}
\dot{V}_{1} \\
\dot{V}_{2} \\
\dot{V}_{3}
\end{array}\right)
$$

It should be noted that if transmission line 1-3 is disconnected due to a line fault, the system is no longer observable since bus 3 cannot be observed by the PMU.

\subsection{Dynamic Observability}

The dynamic model of this system, which is shown as follows, is formulated by classical rotor dynamic equations, where generator rotor angle and rotor speed are the state variables.

$$
\begin{aligned}
& \dot{\delta}_{1}=\omega_{1} \\
& M_{1} \dot{\omega}_{1}=P_{M 1}-\frac{E_{1} E_{2}}{X_{12}} \sin \left(\delta_{1}-\delta_{2}\right) \\
& -\frac{E_{1} E_{3}}{X_{13}} \sin \left(\delta_{1}-\delta_{3}\right)-D_{1} \omega_{1} \\
& \dot{\delta}_{2}=\omega_{2} \\
& M_{2} \dot{\omega}_{2}=P_{M 2}-\frac{E_{1} E_{2}}{X_{12}} \sin \left(\delta_{2}-\delta_{1}\right) \\
& -\frac{E_{2} E_{3}}{X_{23}} \sin \left(\delta_{2}-\delta_{3}\right)-D_{2} \omega_{2} \\
& \dot{\delta}_{3}=\omega_{3} \\
& M_{3} \dot{\omega}_{3}=P_{M 3}-\frac{E_{1} E_{3}}{X_{13}} \sin \left(\delta_{3}-\delta_{1}\right) \\
& -\frac{E_{2} E_{3}}{X_{23}} \sin \left(\delta_{3}-\delta_{2}\right)-D_{3} \omega_{3}
\end{aligned}
$$

The parameters of the 3-generator system are given in [1]. Assume there is a three-phase fault followed by normal clearing on transmission line 1-3. During the fault, the dynamical system in (20) is changed since branch 1-3 is disconnected. Then it is assumed that the breaker recloses successfully at $1 \mathrm{~s}$ and the system topology is recovered.

Assume that there is one PMU at bus 1 and the corresponding states of $\delta_{1}$ and $\omega_{1}$ are directly monitored. By implicit integration method with trapezoidal rule, the states can be calculated by PMU measurement [1]. The result is shown in Fig. 2.

The observability of system is calculated along the dynamic trajectory. The smallest singular value of the observability matrix which serves as an index for the level of observability of the nonlinear dynamic system is shown in Fig. 3.
As seen from the figure, the level of observability changes over time due to the change of the system states where observability is evaluated. For most of the time, the smallest singular values along the trajectory are greater than zero, indicating that the system is almost observable except for a few snapshots. However, the smallest singular values are always close to zero and, therefore, the system is poorly observable. Due to the measurement noises, modeling errors and uncertainties, system can lose its observability over time.

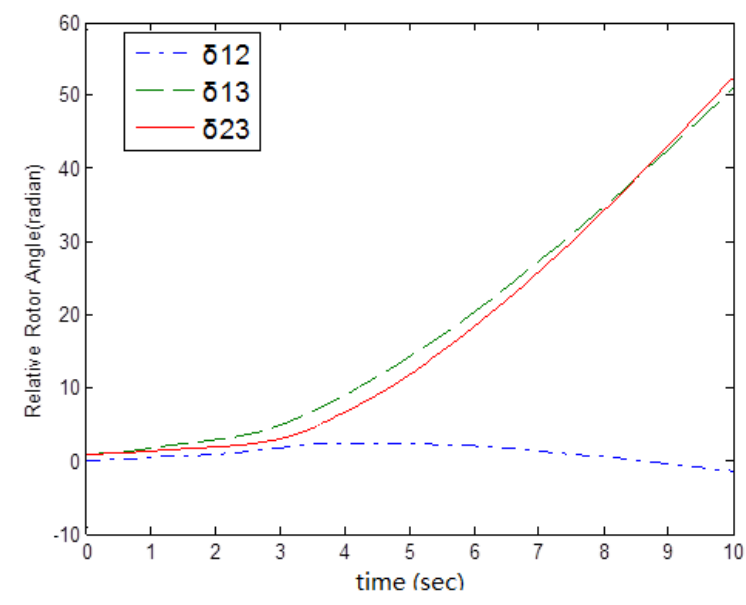

Figure 2 Simulation results of relative rotor angles



Figure 3 Smallest singular value of observability matrix with PMU placed at bus 1

For various cases in which PMUs are placed at bus 1 and 2 , or at all three buses, the smallest singular values of the observability matrix along the solution are shown in Fig. 4 and Fig. 5. The minimum and maximum singular values within the 10-second interval for the three cases are shown in Table 1. The results clearly indicate that when the number of PMUs is small, the system will have a lower level of observability. When the numbers of PMUs increases, the level of observability is also improved. 
This is an expected result as the power grids continue to increase the number of PMUs on the systems.

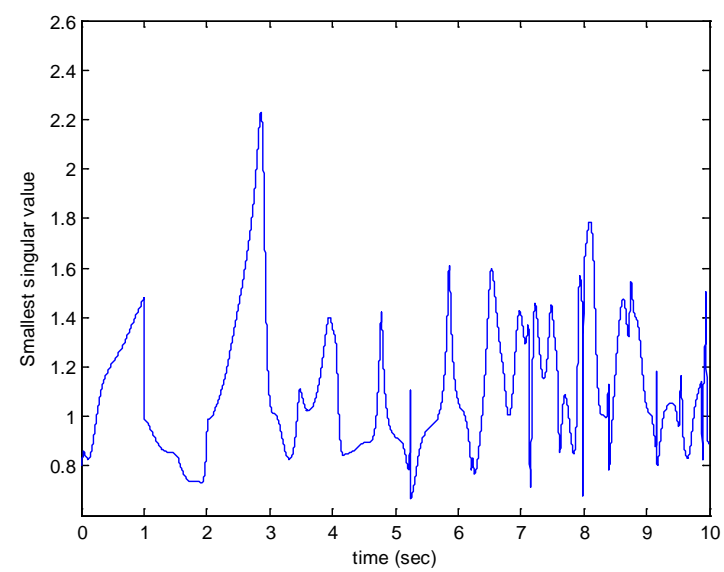

Figure 4 Smallest singular value of observability matrix with PMUs placed at bus 1 and 2

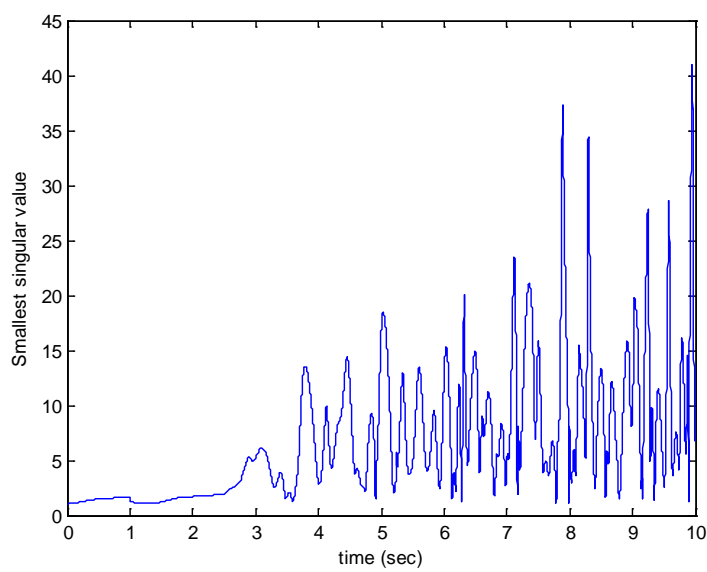

Figure 5 Smallest singular value of observability matrix with PMUs placed at all buses

Table 1 The minimum and maximum singular values within the 10second interval for three cases

\begin{tabular}{|c|c|c|}
\hline & Minimum value & Maximum value \\
\hline PMU at bus 1 & $1.2910 \mathrm{e}-006$ & 0.06348 \\
\hline PMUs at bus 1 and 2 & 0.6686 & 2.227 \\
\hline PMUs at all buses & 1.0111 & 40.95 \\
\hline
\end{tabular}

\section{Conclusions}

The proposed observability formulation provides a systematic way to indicate how much information can be extracted from the limited available measurements, i.e., PMU data. In the dynamic case, the concepts of solvability and observability are separated from each other. With a higher level of observability, the dynamic states reconstructed from PMU measurement are more reliable. Since dynamic state estimation can be performed with PMU measurements, the proposed method is a contribution toward an on-line application for PMUs for wide area monitoring and control to improve the dynamic performance of power systems.

\section{References}

[1] J. Yan, C. C. Liu, and U. Vaidya, "PMU Based Monitoring of Rotor Angle Dynamics,” IEEE Transactions on Power Systems, vol. 26, no. 4, pp. 2125-2133, 2011.

[2] A. G. Phadke, J. S. Thorp, and K. J. Karimi, "State Estimation with Phasor Measurements," IEEE Transactions on Power Systems, vol. 1, no. 1, pp. 233-238, 1986.

[3] B. Gou, "Generalized Integer Linear Programming Formulation for Optimal PMU Placement”, IEEE Transactions on Power Systems, vol. 23, no. 3, 2008.

[4] C. J. Dafis, "An Observability Formulation for Nonlinear Power Systems Modeled as Differential Algebraic Systems,” Ph.D. dissertation, the Center for Electric Power Engineering, Drexel University, Philadelphia, PA.

[5] A. Monticelli and F. F. Wu, "Network Observability: Theory," IEEE Transactions on Power Apparatus and Systems, vol. 104, no. 5, pp. 1042-1048, 1985.

[6] A. Abur and A. G. Exposito, Power System State Estimation: Theory and Implementation, Marcel Dekker, 2004.

[7] M. Nakhaeinejad and M. D. Bryant, "Observability Analysis for Model-Based Fault Detection and Sensor Selection in Induction Motors”, Measurement Science and Technology, vol. 22, no. 7, pp. 075202, 2011.

[8] F. C. Schweppe, J. Wildes and D. B. Rom, "Power System Static State Estimation, Part I, II, III", IEEE Transactions on Power Apparatus and Systems, vol. 89, no. 1, pp. 120-135, 1970.

[9] A. Monticelli, "Electric Power System State Estimation," Proceedings of the IEEE, vol.88, no. 2, pp. 262-282, 2000.

[10] A. Monticelli and F. F. Wu, "Network Observability: Identification of Observable Islands and Measurement Placement," IEEE Transactions on Power Apparatus and Systems, vol. 104, no. 5, pp. 1042-1048, 1985.

[11] S. Chakrabartiand and E. Kyriakides, "Optimal Placement of Phasor Measurement Units for Power System Observability”, IEEE Transactions on Power Systems, vol. 23, no. 3, pp. 1433-1440, 2008.

[12] R. F. Nuqui and A. G. Phadke, "Phasor Measurement Unit Placement Techniques for Complete and Incomplete Observability,” IEEE Transactions on Power Delivery, vol. 20, no. 4, pp. 2381-2388, 2005.

[13] R. E. Kalman, "On the Computation of the Reachable/Observable Canonical Form”, SIAM Journal of Control and Optimization, Vol. 20, pp. 258-260, 1982.

[14] R. Hermann and A. J. Krener, "Nonlinear Controllability and Observability", IEEE Transactions on Automatic Control, vol. 2, No.5, pp. 728-740, 1977.

[15] K. Röbenack and K. J. Reinschke. "An Efficient Method to Compute Lie Derivatives and the Observability Matrix for Nonlinear Systems", Proc. 2000 International Symposium on Nonlinear Theory and its Applications. Dresden, vol. 2, pp. 625628, 2000

[16] C. T. Chen, Linear System Theory and Design (3rd Edition), Oxford University Press, USA, 1998.

[17] E. Ghahremani and I. Kamwa. "Dynamic State Estimation in Power System by Applying the Extended Kalman Filter With Unknown Inputs to Phasor Measurements”, IEEE Transactions on Power Systems, vol. 26, No. 4, pp. 2556-2566, 2011. 